\title{
Studies on Microdrilling of NbN Superconductor using Pulsed Laser Ablation
}

\author{
Manikandan Esakkimuthu ${ }^{1}$ and Karthigeyan Arumugam ${ }^{2}$ \\ ${ }^{1}$ Department of ECE, B.S, Abdur Rahman Crescent Institute of Science and Technology, Chennai, India \\ E-mail: manikandane@crescent.education \\ ${ }^{2}$ Department of ECE, SSN College of Engineering, Chennai, India
}

\begin{abstract}
Superconducting materials are of importance in making highly efficient terahertz components for space applications. The creation of microstructure using laser ablation process was provided. A nanosecond pulsed Nd: YAG laser with the maximum energy of $0.6 \mathrm{~J}$ was utilized to make microscale features in $\mathrm{NbN}$ superconducting material. The material was ablated at different energy levels and the corresponding spectra were captured with spectrometer through fiber optic cable. The exact energy required to ablate the $\mathrm{NbN}$ material was obtained experimentally. The experimental part utilizes a $1064 \mathrm{~nm}$ ns-pulsed laser as a source for ablation. The same process has been repeated in high-pressure conditions of $4 \mathrm{~atm}$ and $8 \mathrm{~atm}$ in a vacuum chamber. The ablation rate was decreased as the pressure level in the vacuum chamber has been increased; solidification of molten material was observed under high-pressure conditions. The effects of laser intensity and scanning speed in the ablation rate of $\mathrm{NbN}$ material have been experimentally verified. The poor thermal conductivity of the $\mathrm{NbN}$ makes the nanosecond laser ablation as an effective process for micromachining with minimal heat affected area. The morphological properties of the ablated structure were evaluated using optical microscopic images.
\end{abstract}

DOI: 10.2961/jlmn.2019.03.0004

Keywords: terahertz, superconductor, laser ablation, microstructure, pressure

\section{Introduction}

In recent years, the utilization of unexplored $\mathrm{THz}$ spectrum drives many industries (security, medical, telecommunication, space, pharmaceutical, chemical etc) after the successful developments of high power sources for the generation [1], THz-time domain spectroscopy (TDS) technique, high sensitive detectors etc. This motivates us to develop many $\mathrm{THz}$ devices include frequency selective surface (FSS) filter for earth observation systems [2], metamaterials for manipulating $\mathrm{THz}$ waves [3-5], antennas for detecting terahertz wave [6], THz modulator [7] and so forth. Two major issues should be addressed in developing these devices for terahertz applications. First, the metal (copper) has lossy nature at higher frequencies i.e. towards terahertz frequencies is prominent which gives the negative impact on the performance of the systems. The second consideration is the size of the device is comparable to the wavelength i.e. in the order of micron scale, so creating microstructure is an essential and difficult task [8]. The first issue could be resolved by using low loss superconducting materials for device fabrication.

Recently, terahertz metamaterials (MM) made up of the superconducting film has been reported in the literature [9]. Initially, high-temperature superconducting material example, yttrium-barium-copper-oxide (YBCO) has been utilized in making THz MM, but it has the same issue, as the surface resistance is comparable to the copper at the critical temperature in the $\mathrm{THz}$ band. So MM based on niobium nitride $(\mathrm{NbN})$ was chosen as an effective alternative which provides lower resistance than YBCO film [10]. The second major issue is in terms of micro-structuring thin films for device fabrication. Many lithography techniques are available for fabricating microscale components such as
UV lithography, E-beam technique, imprint technology etc. but these require highly expensive equipments, clean room environments and also a time-consuming process. Currently, researchers have used photolithography and reactive ion etching techniques to pattern the superconducting material. Laser ablation could provide an alternative solution and easy way of making microstructures in a variety of materials because of its unique nature such as highly coherent and monochromatic, high-intensity flux etc. With the developments of short and ultra-short pulses, micro-components can be fabricated effectively with high resolution $[11,12]$.

Nanosecond laser ablation is a kind of thermal process where the material is removed from the substrate by means of melting and vaporization [13]. Previously many numerical and experimental investigations for ns-pulsed laser ablation of different materials like copper, ITO, dielectrics, ceramics, steel have been reported in the literature [14-16].

The first time, we report on the ns-pulsed laser ablation $\mathrm{NbN}$ superconductor deposited on a magnesium oxide ( $\mathrm{MgO})$ substrate by reactive dc magnetron sputtering. In this work, the effect of laser fluence on the ablation process at constant scanning speed and repetition rate has been reported for a single shot of laser pulse. The characterization of the results includes optical microscopic images, depth measurement using non-contact optical profilometer and a spectrum obtained during ablation by the spectrometer.

\section{Experimental Studies}

This section introduces the deposition of an $\mathrm{NbN}$ thin film by sputtering followed by the nanosecond laser ablation. 


\subsection{Deposition}

An epitaxial thin film of $\mathrm{NbN}$ was deposited on (100) oriented magnesium oxide $(\mathrm{MgO})$ substrate by reactive dc magnetron sputtering. The thin film $\mathrm{NbN}$ was deposited by sputtering by keeping the $\mathrm{N}_{2} / \mathrm{Ar}$ ratio at $(20: 80)$ while maintaining the substrate temperature and pressure at 600 ${ }^{\circ} \mathrm{C}$ and $6.58 \mu \mathrm{atm}$ respectively. The thickness of the deposited $\mathrm{NbN}$ film and the substrate was $200 \mathrm{~nm}$ and $0.5 \mathrm{~mm}$ respectively. A detailed procedure for the deposition and measurement of superconductivity properties of $\mathrm{NbN}$ was reported in the previous work [17].

\subsection{Material Optical Properties}

In this work, nanosecond laser operating at $1064 \mathrm{~nm}$ wavelength has been utilized. So it is necessary to obtain the optical properties at a particular wavelength. So the reflectivity and transmission characteristics of the $\mathrm{NbN}$ deposited on $\mathrm{MgO}$ substrate was done using UVvisible/NIR Spectrophotometer (JASCO V-770) wavelength ranging from $190 \mathrm{~nm}-2700 \mathrm{~nm}$. The absorption coefficient was obtained from the transmission and reflectivity characteristics at $1064 \mathrm{~nm}$ using Beer-Lambert law. $\alpha(\lambda)=\left(-\frac{1}{t} \ln \left[T(\lambda) /\left\{\left(1-R^{\prime}(\lambda)\right)(1-R(\lambda))\right\}\right]\right)$

Where $t$ is the thickness of the sample, $T(\lambda)$ and $R(\lambda)$ is the measured transmission and reflectivity of the air $/ \mathrm{NbN}$ interface, $R^{\prime}(\lambda)$ is the calculated reflectivity of the $\mathrm{NbN} / \mathrm{MgO}$ interface and the value is given by equation (2). $R^{\prime}(\lambda)=\left(\left(\eta_{N b N}-\eta_{M g O}\right)\left(\eta_{N b N}+\eta_{M g O}\right)\right)^{2}$

In equation (2), $\eta$ is the refractive index of $\mathrm{NbN}$ and $\mathrm{MgO}$ material which are given as 2.09 and 1.7 respectively. The resultant reflectivity of $\mathrm{NbN} / \mathrm{MgO}$ interface at $1064 \mathrm{~nm}$ is 0.01. The calculated optical constants are listed in Table 1.

\subsection{Laser Ablation}

A thin film $\mathrm{NbN}$ of $10 \mathrm{~mm} \times 10 \mathrm{~mm}$ was used as a sample. The ablation setup uses the 7 ns-pulsed laser operating at the wavelength of $1064 \mathrm{~nm}$ as a source (model no-Nd: YAG Laser-704G-10). The maximum energy supplied by the laser source is $0.6 \mathrm{~J}$ with a repetition rate of $10 \mathrm{~Hz}$. The energy supplied to the target material during ablation process was measured by a joule meter (QE 50LP-S-MB-D0) which has the sensitivity of $3 \mathrm{~J} / \mathrm{V}$ with a maximum measurable value up to $85 \mathrm{~J}$. The corresponding voltage level is viewed in a digital storage oscilloscope (DSO). The plasma generated in ablation process is captured with the help of collimator which is fed into the spectrometer through fibre optic cable (FOC). The core diameter and the numerical aperture of the FOC (BFH3T-600) are 600 microns and 0.39 , respectively. The spectrometer (MCS $55 \mathrm{~m} 8 \mathrm{MA} / \mathrm{UV}-$ NIR) wavelength falls in the region between (190-1015) $\mathrm{nm}$ with a resolution of $1 \mathrm{~nm}$ and the obtained spectrum is displayed in the computer. The schematic diagram of the laser ablation setup with the high-pressure chamber and the spectrometer was shown in Fig.1. The sample is kept in a target holder which is located inside the chamber and the high-pressure conditions are maintained by the highpressure pump. The laser light then hit the target through the quartz window located on the front side of the chamber for ablation process. On completing the experiments the pressure levels can be reduced to the atmospheric value by releasing it through the exit valve. The ablation depth was analyzed offline using a non- contact optical profilometer (BRUKER, ConourGTInMotion).

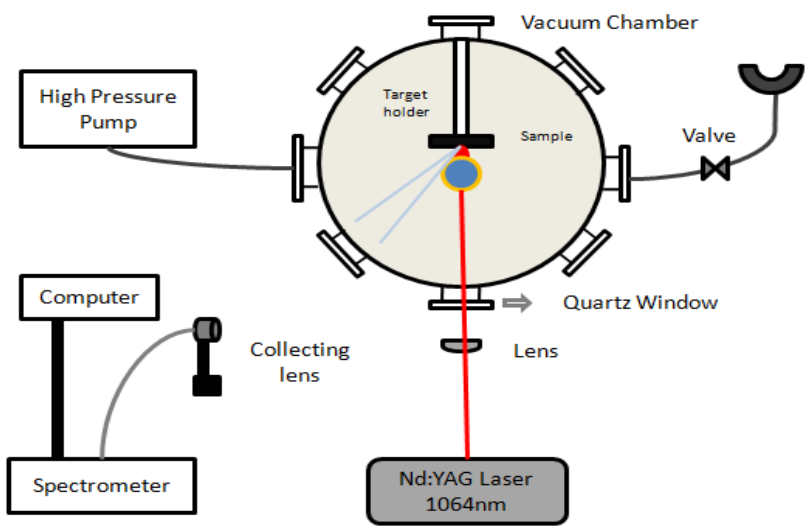

Fig.1 Schematic diagram of the setup used for laser ablation of $\mathrm{NbN}$ with high-pressure chamber

\section{Results and Discussions}

The superconductivity nature of a deposited $200 \mathrm{~nm}$ thin film $\mathrm{NbN}$ material was obtained by using obtaining 2 coil AC susceptibility measurements. The corresponding result was shown in Fig 2(a). From the figure it was observed that the critical temperature of the film was $15.5 \mathrm{~K}$. The real $(\mathrm{Vx})$ and imaginary parts $(\mathrm{Vy})$ of the susceptibility were shown in the figure.
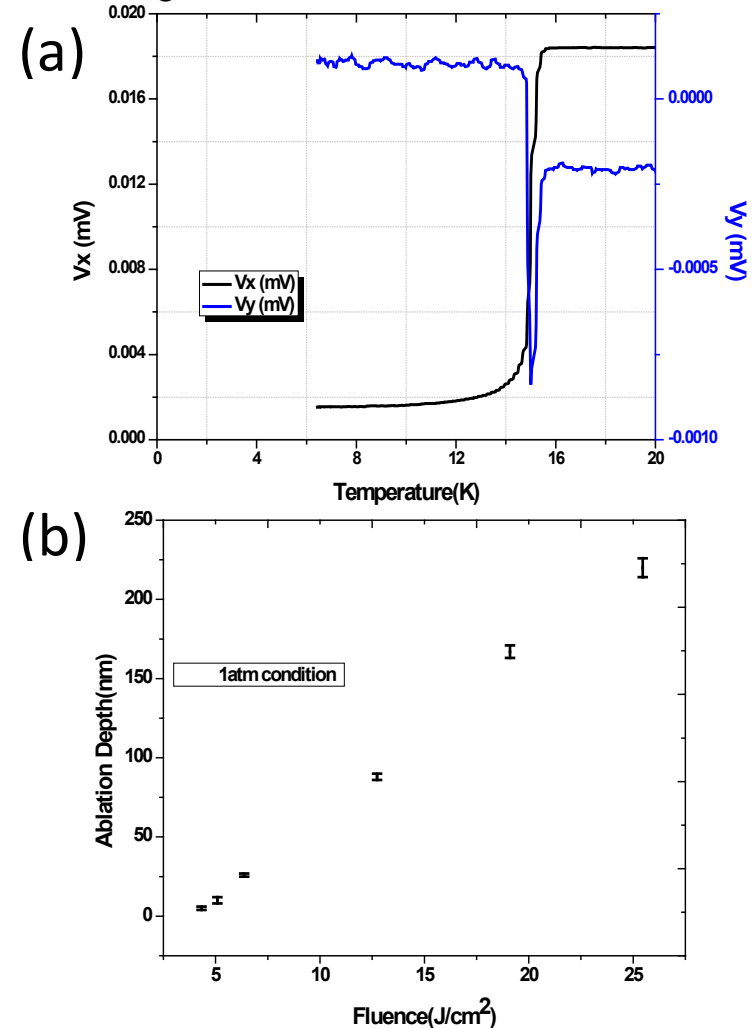

Fig.2 (a) 2 coil AC susceptibility measurement of $200 \mathrm{~nm}$ $\mathrm{NbN}$ on $\mathrm{MgO}$, observed Tc $15.5 \mathrm{~K}$. (b) Effect of nanosecond pulsed laser fluence on the ablation depth of $\mathrm{NbN}$ at ambient condition. 

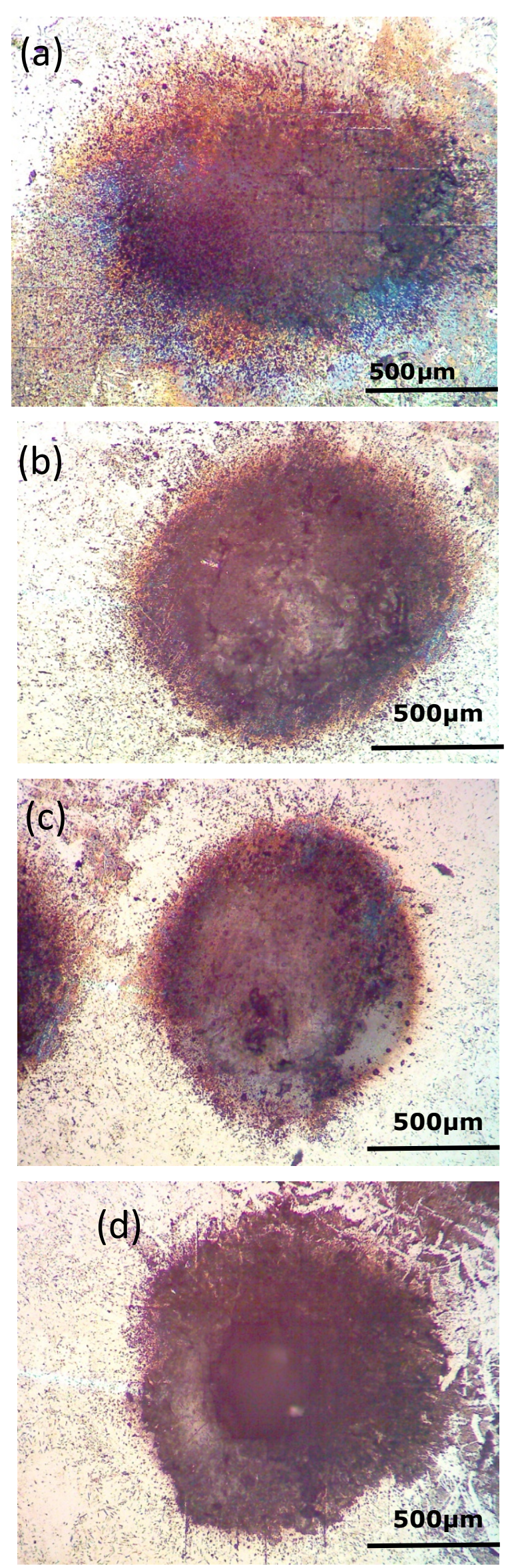
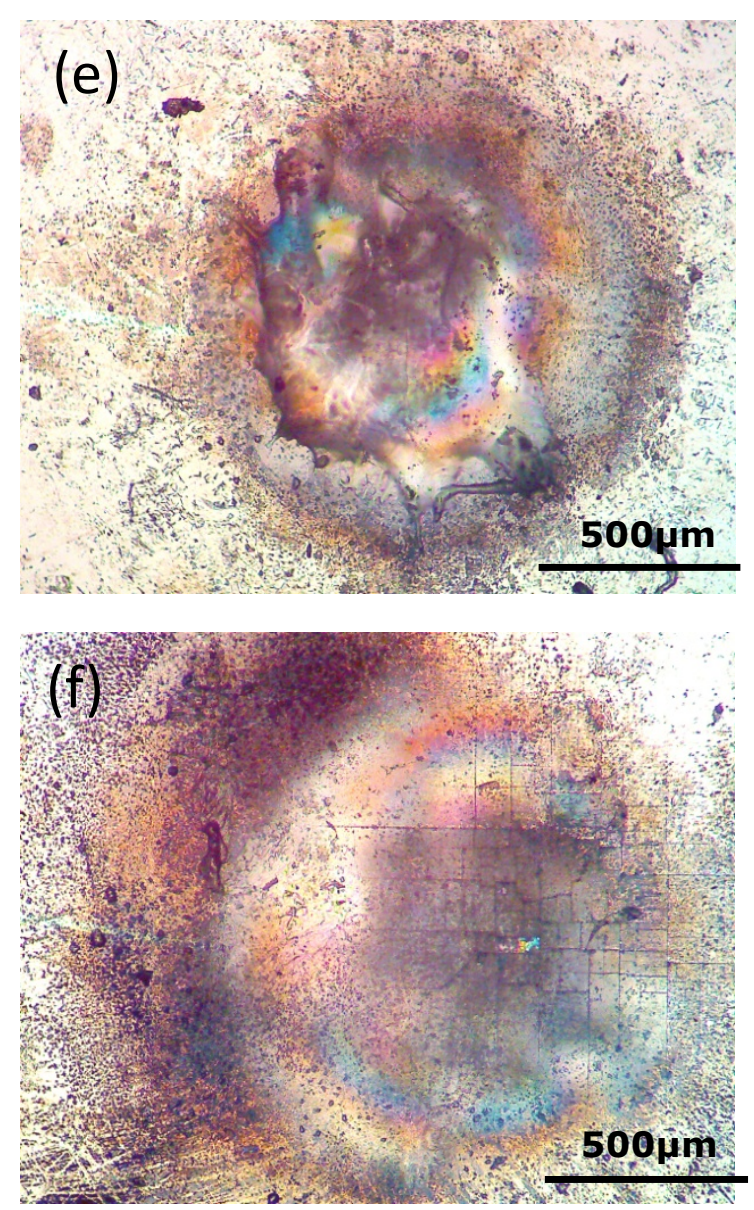

Fig.3 Optical microscopic images of the ablated NbN. In all the cases the diameter of the image is approximately $1 \mathrm{~mm}$ including the heat affected zone (HAZ) energy supplied (a) $17 \mathrm{~mJ}\left(4.33 \mathrm{~J} / \mathrm{cm}^{2}\right)$, (b) $20 \mathrm{~mJ},\left(5.09 \mathrm{~J} / \mathrm{cm}^{2}\right)$, (c) $25 \mathrm{~mJ},\left(6.37 \mathrm{~J} / \mathrm{cm}^{2}\right)$, (d) $100 \mathrm{~mJ},\left(25 \mathrm{~J} / \mathrm{cm}^{2}\right)$, (e) $100 \mathrm{~mJ}$ at $4 \mathrm{~atm}$, and (f) $100 \mathrm{~mJ}$ at $8 \mathrm{~atm}$ condition.

Laser ablation is a process of removing the material by means of vaporization. When the laser hits the target metal, the energy is absorbed by the free electrons first by inverse bremsstrahlung. Then the absorbed energy is converted to lattice vibrations via electron-phonon coupling mechanism on a few picoseconds timescale. Therefore the nanosecond laser ablation is a thermal process for metallic targets. When the laser fluence is greater than the threshold fluence of the material melting takes place. After melting, vaporization occurs. At higher fluence levels, the vapour plume formation occurs which consist of the ionized particles. The emission from the ionized particles was captured by the collecting lens and transferred to the spectrometer through fiber optic cable. Thus the ionized particles of the ablated sample were seen as the spectrum.

The effect of laser intensity on the ablation depth of $\mathrm{NbN}$ material is shown in Fig.2 (b). It was found that the threshold fluence of ns-pulsed ablation of $\mathrm{NbN}$ is $4.33 \mathrm{~J} / \mathrm{cm}^{2}$. The corresponding ablation depths for different fluence values along with their corresponding error values are depicted.

The optical microscopic images of the laser ablated samples were shown in Fig.3. Initially, the sample was treated 
with the energy of $2 \mathrm{~mJ}$. Before analyzing the depth characteristic, and the corresponding spectrum of the laser treated sample was captured with the help of spectrometer from the plasma. No ablation was started in an energy range of from $2 \mathrm{~mJ}$ to $17 \mathrm{~mJ}$. When the energy of the laser source was kept at $17 \mathrm{~mJ}$ an ablation process started which was confirmed by obtaining the plasma captured by the spectrometer. The microscopic images of the ablated $\mathrm{NbN}$ sample at $17 \mathrm{~mJ}, 20 \mathrm{~mJ}$ and $25 \mathrm{~mJ}$ are shown in Fig. 3 (a), (b) and (c) respectively. It is observed that a minimum of $17 \mathrm{~mJ}$ energy is required to ablate the $\mathrm{NbN}$ superconducting thin film for the ns-pulsed laser. The laser beam was excited to hit the same place at $20 \mathrm{~mJ}$ and the corresponding spectrum was shown in Fig.4 (a).

At $100 \mathrm{~mJ}$ of energy with 1 atm condition, a clear hole was obtained and it is shown in Fig. 3 (d) and the corresponding spectrum is depicted in Fig. 4 (a). It shows that a higher amount of energy was required to remove the entire $\mathrm{NbN}$ material from the substrate by laser ablation process. The reason for this higher energy was the melting point of the $\mathrm{NbN}$ is $2846 \mathrm{~K}$ comparatively very higher in value than the normal metals. At high-pressure conditions by theory, the thermal conductivity of the material goes on decreasing which provides the support for ablating material with a minimal heat affected area. And in the case of nanoparticles generation ablation at high pressure and high-density media is preferable where the sizes of the particles are easily controllable.

In this work the ablation process has been studied for high-pressure environmental conditions and the results are also obtained experimentally. But at higher pressure, the ablation takes place in a slow manner which also requires a more amount of energy to ablation occurs. The Figs.3 (e) and (f) corresponds to the optical microscopic images for 4 atm and $8 \mathrm{~atm}$ pressure conditions with $100 \mathrm{~mJ}$ incident energy. This process of ablation started at $100 \mathrm{~mJ}$ of energy which is comparatively higher to the ablation occurs at atmospheric pressure. As the pressure keeps on increasing from 1 atm to $8 \mathrm{~atm}$ the amount of redeposition is high which happened because of lag in the energy supplied. This develops the solidification of molten material during ablation.

The corresponding spectrum captured by the spectrometer was shown in Fig.4 (b). It was observed that the intensity level was very low then the ablation at $20 \mathrm{~mJ}$ energy in the atmospheric environment. Fig.3 (c) shows the optical image of the ablated pattern at $100 \mathrm{~mJ}$ of energy in the atmospheric environment and it was seen that a clear hole has been obtained. This develops a micro hole in the $\mathrm{NbN}$ thin films. This represents the minimum amount of energy required to ablate the $\mathrm{NbN}$ as $17 \mathrm{~mJ}$ which corresponds to the fluence of $4.33 \mathrm{~J} / \mathrm{cm}^{2}$ for the spot size of approximately $1 \mathrm{~mm}$. The $\mathrm{Mg}$ ions start to appear in the spectrum and were identified at the wavelength of $309 \mathrm{~nm}$ for $100 \mathrm{~mJ}$ of energy. This indicates that the $\mathrm{NbN}$ was completely etched away from the substrate. The elemental decomposition during the laser ablation was observed by the spectrometer. The captured spectra were compared with the NIST reference database and marked in the spectrum (Fig.4 (a) \& (b)).

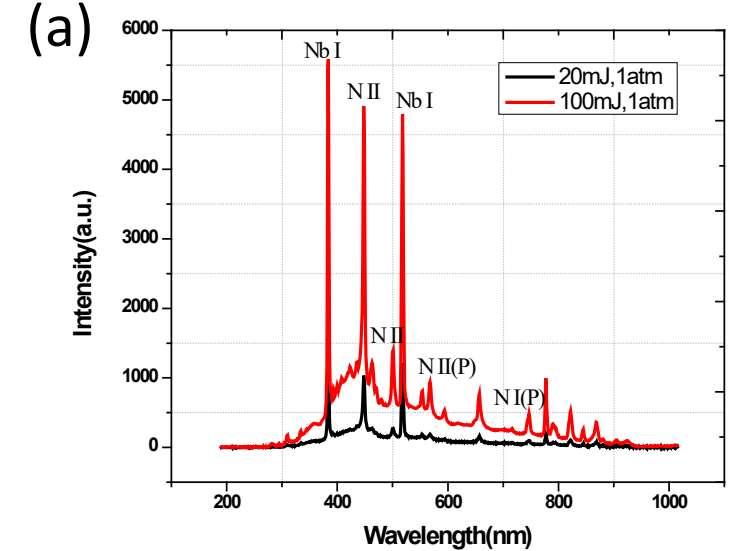

(b)

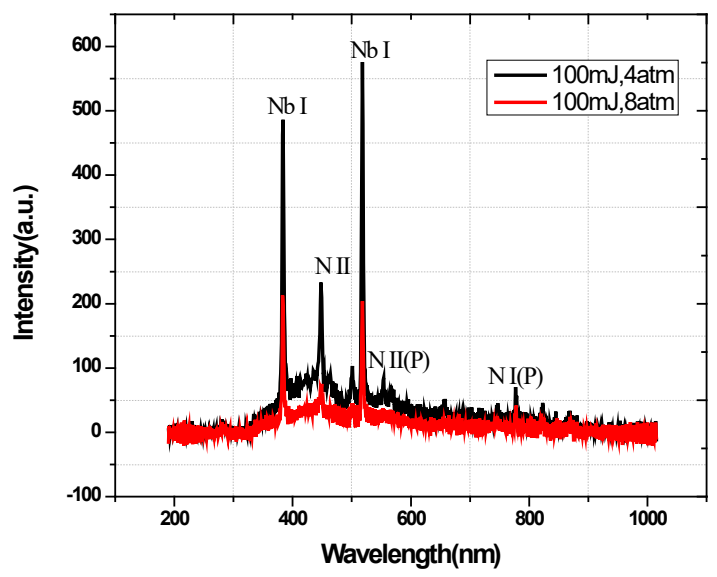

Fig.4 Spectra obtained in laser ablation of $\mathrm{NbN}$ film (a) at two different energies in atmospheric and (b) high pressure environment (identification $\mathrm{Nb}$ and $\mathrm{N}$ ions during ablation are marked in the figure) P-Prominent line, I-First Ionization, II-Second Ionization

The analysis of the results shows that the higher melting point of $\mathrm{NbN}$ makes laser ablation as a challenging task which requires higher fluence than the normal metals. At higher pressure, the coefficient of thermal expansion value is getting decreased further which makes the decrease in the ablation area compared to the normal atmosphere. This phenomenon leads to decrease in intensity level of individual elements obtained in the spectra. The observation was, by supplying high amount energy to the material the desired structure and depth could be obtained with minimal heat affected area at high-pressure conditions.

The damage threshold in $\mathrm{NbN}$ material for the applied laser fluence can be calculated by using the linear relationship equation between the natural logarithmic of the applied energy and the crater diameter as,

$$
D^{2}=2 d^{2} \ln \left(\frac{E_{0}}{E_{t h}}\right)
$$

Where $D$ is the diameter of the damaged (crater) area, $E_{0}$ is the applied laser fluence, $\mathrm{E}_{\text {th }}$ is the threshold laser fluence and $2 \mathrm{~d}$ is the Gaussian beam spot size. A graphical representation of damage area diameter at various laser fluence for the 7 ns pulsed laser is depicted in Fig.5. This shows that the damage threshold fluence for $7 \mathrm{~ns}$ laser source is $4.33 \mathrm{~J} / \mathrm{cm}^{2}$. 


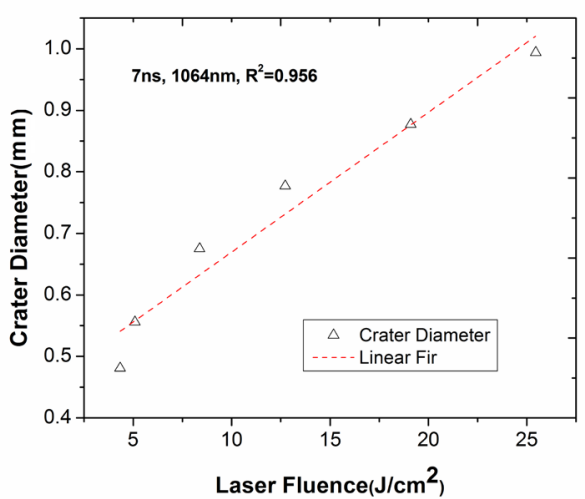

Fig.5 Laser ablated crater diameter for the various applied laser fluence.

The temperature distribution and the peak temperature value for the ablation of $\mathrm{NbN}$ using nanosecond laser source is done numerically using finite element method (FEM) technique. This was done using standard differential heat conduction equation [14]. The optical and the thermal properties of the $\mathrm{NbN}$ are shown in Table $1[18,19]$.

Table $1 \mathrm{NbN}$ thermal properties.

\begin{tabular}{|l|l|}
\hline Properties & Niobium Nitride \\
\hline CTE $\left(10^{-6}\right)\left(1 /{ }^{0} \mathrm{C}\right) @ \mathrm{RT}$ & 10.1 \\
\hline Thermal Conductivity(W/m-K) & 3.35 \\
\hline Specific heat(J/kg-K) & 1.52 \\
\hline Melting Temperature(K) & 2846 \\
\hline Reflectivity R & 0.01 \\
\hline Absorption Coefficient $\alpha\left(\mathrm{m}^{-1}\right)$ & $1.005^{*} 10^{\wedge} 6$ \\
\hline Density $\rho$ & $8470 \mathrm{Kg} / \mathrm{m}^{3}$ \\
\hline Optical absorption length $(\mathrm{nm})$ & 995 \\
\hline Thermal diffusion length(nm) & 13.42 \\
\hline Young's Modulus E(GPa) & 313 \\
\hline Poisson ratio P & 0.23 \\
\hline Thermal diffusivity & $2.6^{*} 10^{\wedge}-4$ \\
\hline
\end{tabular}

It has a higher melting point and poor thermal conducting properties compared to other metals. The incident heat source from the laser not only interacts with the surface of the material but also absorbed by the inner area of the film based on the absorption coefficient value. The absorption of heat energy further evaporates the material when sufficiently enough to vaporize the material and forms the thermal diffusion area in the adjacent places. This can be measured by calculating the thermal diffusion length $\left(l_{T}\right)$.
The thermal diffusion length of $\mathrm{NbN}$ is calculated from the thermal diffusivity $(\kappa)$ and the interaction time $(t)$ by using the below-mentioned formula.

$$
l_{T, N b N} \approx \sqrt{\kappa t}
$$

The peak temperature during the laser ablation was obtained using FEM numerical simulation. It was shown that at the laser fluence of $4.33 \mathrm{~J} / \mathrm{cm}^{2}$, the temperature at the surface of $\mathrm{NbN}$ was near to its melting point at $10 \mathrm{~ns}$ for the $1064 \mathrm{~nm}$ operating wavelength. Further supplied laser energy helped in vaporizing the material from the substrate and the material was completely removed at $0.1 \mathrm{~J}$.

\section{Conclusions}

Nanosecond laser ablation of thin film $\mathrm{NbN}$ superconductor deposited on an $\mathrm{MgO}$ substrate has been reported in this paper. The threshold fluence required to ablate the material with a $7 \mathrm{~ns}$ laser operating at $1064 \mathrm{~nm}$ wavelength was obtained experimentally. The heat affected area during the nanosecond laser ablation process was comparatively less than occurred in other metals because of low thermal conductivity nature of $\mathrm{NbN}$. The ablation process has been repeated in a high-pressure chamber at 4 atm and 8 atm levels. It was observed that large amount of energy almost 5 times higher than 1 atm should be supplied at high pressure for the ablation. The characterization of the results includes optical microscopic images, depth measurement using noncontact profilometer and spectrometer analysis. The ablation process has been done at constant scanning speed and repetition rate. The surface characteristics and ionized stage of the ablated structure were evaluated using optical microscopic images and spectrometer. The ablation threshold fluence was fund to be $4.33 \mathrm{~J} / \mathrm{cm}^{2}$ in the atmospheric environment.

\section{Acknowledgement}

The authors thank Prof. Pratap Raychaudhuri, superconductivity lab, TIFR and Dr.Sathiesh Kumar, Department of ECE, MIT, Chennai for providing samples and helping in experimental part.

\section{References}

[1] M.Tonouchi: Nat. Photonics. , 1, (2007) 97.

[2] R.Dickie, R.Cahill, V.Fusco, H. Gamble, and N. Mitchell: IEEE T THz Sci Techn. , 1, (2011) 450.

[3] Hou-Tong Chen, Willie J. Padilla, M. O.Joshua Zide, C. Arthur Gossard, J.Antoinette Taylor and Richard D. Averitt: Nature, 444, (2006) 597.

[4] E. Manikandan, B.S. Sreeja, S. Radha and Ravi Nathuram Bathe: Mater Lett. , 229, (2018) 320.

[5] E. Manikandan, B. S. Sreeja, S. Radha, Ravi Nathuram Bathe, Ravikumar Jain and ShriganeshPrabhu: Int J Infrared Milli, 40, (2018) 38.

[6] D. Palaferri, Y. Todorov, Y. N. Chen, J. Madeo, A. Vasanelli, L. H. Li, A. G. Davies, E. H. Linfield, and C. Sirtori: Appl. Phys. Lett. 106, (2015) 161102.

[7] P. K. Singh, and S. Sonkusale: Sci. Rep. 7, (2017) 40933.

[8] ManikandanEsakkimuthu, B.S.Sreeja, S.Radha, Abhishek Gupta and Goutam Rana, S.Prabhu: JLMN- J Laser Micro Nanoen, 12, (2017) 313. 
[9] Jianqiang Gu,Ranjan Singh,Zhen Tian,Wei Cao Qirong Xing, Mingxia He, Jingwen W. Zhang, Jiaguang Han, Hou-Tong Chen, and Weili Zhang: Apply Phys Lett. , 97, (2010) 071102.

[10]Biaobing Jin, Caihong Zhang, Sebastian Engelbrecht,Andrei Pimenov,Jingbo Wu,Qinyin Xu,Chunhai Cao, Jian Chen, Weiwei Xu, Lin Kang, and Peiheng Wu: Opt Express.,18, (2010) 17504.

[11] Ursula Keller: Nature, 424, (2003) 831.

[12]E. Manikandan, B. S. Sreeja, S. Radha, MuthukannanDuraiselvam, Abhishek Gupta and Shriganesh Prabhu: Opt Eng., 58, (2018) 011007.

[13]Benxin Wu and Yung C. Shin: J. Appl. Phys., 99, (2006) 084310

[14]Davide Bleiner, David Autrique, Zhaoyang Chen and: Annemie Bogaerts: J. Anal. At. Spectrom, 21, (2006) 910.

[15] Inam Mirza, Nadezhda M. Bulgakova, Jan Tomáštík, Václav Michálek, Ondřej Haderka, Ladislav Fekete and Tomáš Mocek: Sci. Rep. ,6, (2016) 39133.

[16] Vladimir I. Mazhukin, Laser ablation-From fundamentals to applications, (INTECH, 2017), p.31.

[17] Chockalingam, S. Chand, Madhavi, Jesudasan, John, Tripathi, Vikram, Raychaudhuri, Pratap: Phys. Rev. B, 77, (2008) 214503.

[18] https:/www.americanelements.com/niobium-nitride24621-21-4

[19] https://en.wikipedia.org/wiki/Niobium_nitride

(Received: May 19, 2019, Accepted: November 16, 2019) 